\title{
Sentinel inflammatory demyelinating lesions preceding primary CNS lymphoma
}

\section{Lesões inflamatórias desmielinizantes sentinelas precedendo linfoma primário do SNC}

Danielle Mesquita TORRES', Milena Sales PITOMBEIRA1, Igor Bessa SANTIAGO', Gabriela Joca MARTINS', Kellen Paiva FERMON¹, Daniel Gurgel Fernandes TAVORA', Fernanda Martins Maia CARVALHO1,2

A 29-year-old man presented with acute seizures and visual impairment. Brain magnetic resonance imaging (MRI) showed multiple white matter T2-lesions with incomplete peripheral enhancement (Figures 1A to 1C). Considering the hypothesis of acute disseminated encephalomyelitis, intravenous methylprednisolone (IVMP) was administrated with full recovery. Two years later, he presented right-sided weakness. MRI disclosed a new T2-lesion, and spectroscopy suggested a tumefactive inflammatory pattern (Figures 1D to $1 \mathrm{H})$. New extensive cerebrospinal fluid (CSF) and blood workup, including aquaporin-4-IgG, was unremarkable. Partial improvement was observed following IVMP. Six months later, after new weakness in the left arm along with a new periventricular lesion (Figures 1 I to 1L), a brain biopsy was performed. Histopathological analysis revealed primary central nervous system (CNS) lymphoma (Figure 2) $)^{1,2,3}$.
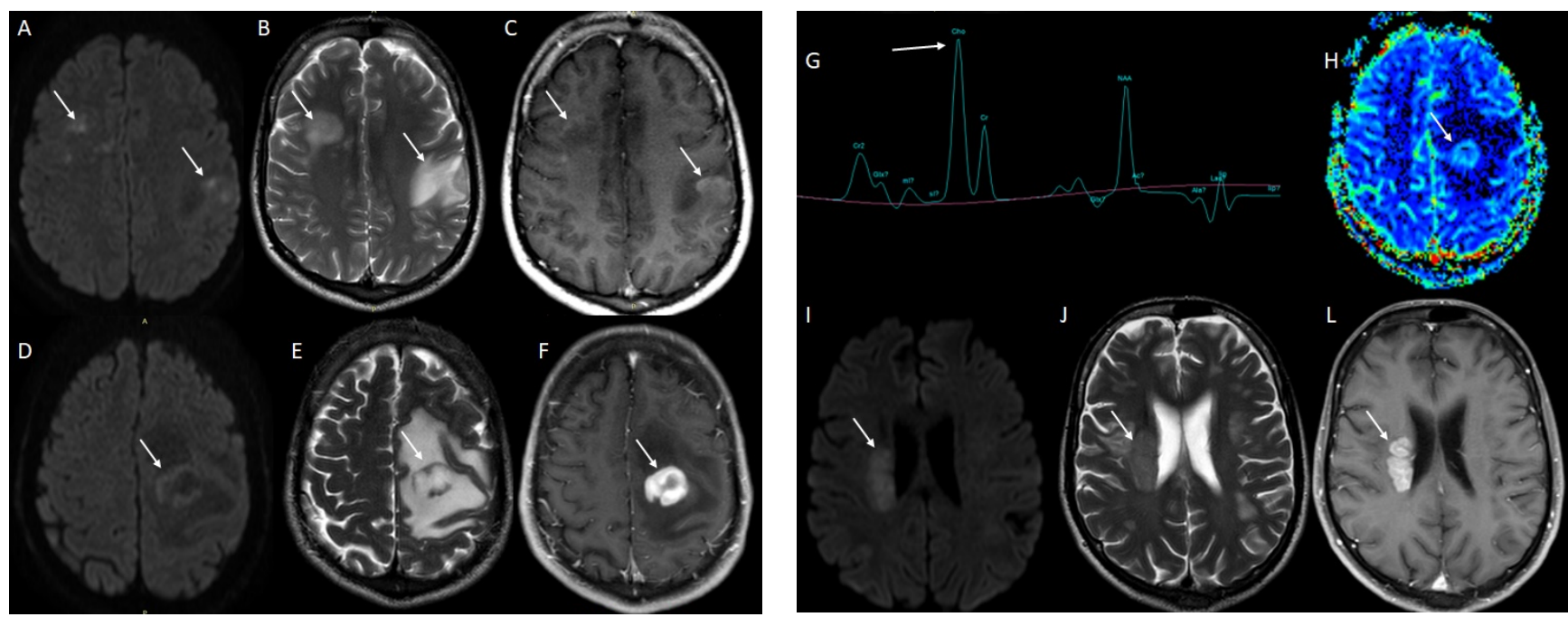

Figure 1. Magnetic resonance imaging exams. (A-C): first magnetic resonance imaging performed on March 2016 indicated diffusion restriction on diffusion-weighted image (A), T2 hypersignal (B), and peripheral enhancement on post-contrast T1 sequences (C). D-H: Neuroimaging performed on July 2018 showed a new lesion with peripheral restricted diffusion on diffusionweighted image (D), T2 hypersignal (E), thick annular enhancement (F), spectroscopy revealed Cho peak increase (G) and minimal relative cerebral blood volume map (rCBV) increase. (H). (I-L): Brain magnetic resonance imaging performed on January 2019 disclosed a new right periventricular lesion with diffusion restriction on diffusion-weighted image (I), mild T2 hyperintensity sequences (J), and homogeneous contrast enhancement (L).

\footnotetext{
${ }^{1}$ Hospital Geral de Fortaleza, Serviço de Neurologia, Fortaleza CE, Brazil.

¿Universidade de Fortaleza, Programa de Pós Graduação em Ciências Médicas, Fortaleza CE, Brazil.

Danielle Mesquita TORRES (D) https://orcid.org/0000-0002-0940-2757; Milena Sales PITOMBEIRA (D) https://orcid.org/0000-0002-3298-0264; Igor Bessa SANTIAGO (ID) https://orcid.org/0000-0002-6408-2186; Gabriela Joca MARTINS (ID) https://orcid.org/0000-0001-8063-1600; Kellen Paiva FERMON (D) https://orcid.org/0000-0002-4251-6430; Daniel Gurgel Fernandes TAVORA (D) https://orcid.org/0000-0002-0570-9815; Fernanda Martins Maia CARVALHO (iD https://orcid.org/0000-0001-6548-7268

Correspondence: Milena Sales Pitombeira; E-mail: milenaspitombeira@gmail.com

Conflict of interest: There is no conflict of interest to declare.

Authors' contributions: DT: literature review, manuscript and imaging preparation, IS: literature review, manuscript preparation; MSP, GJ, KF and FMM: manuscript supervision and revision.

Received on September 4, 2020; Accepted on September 25, 2020.
} 


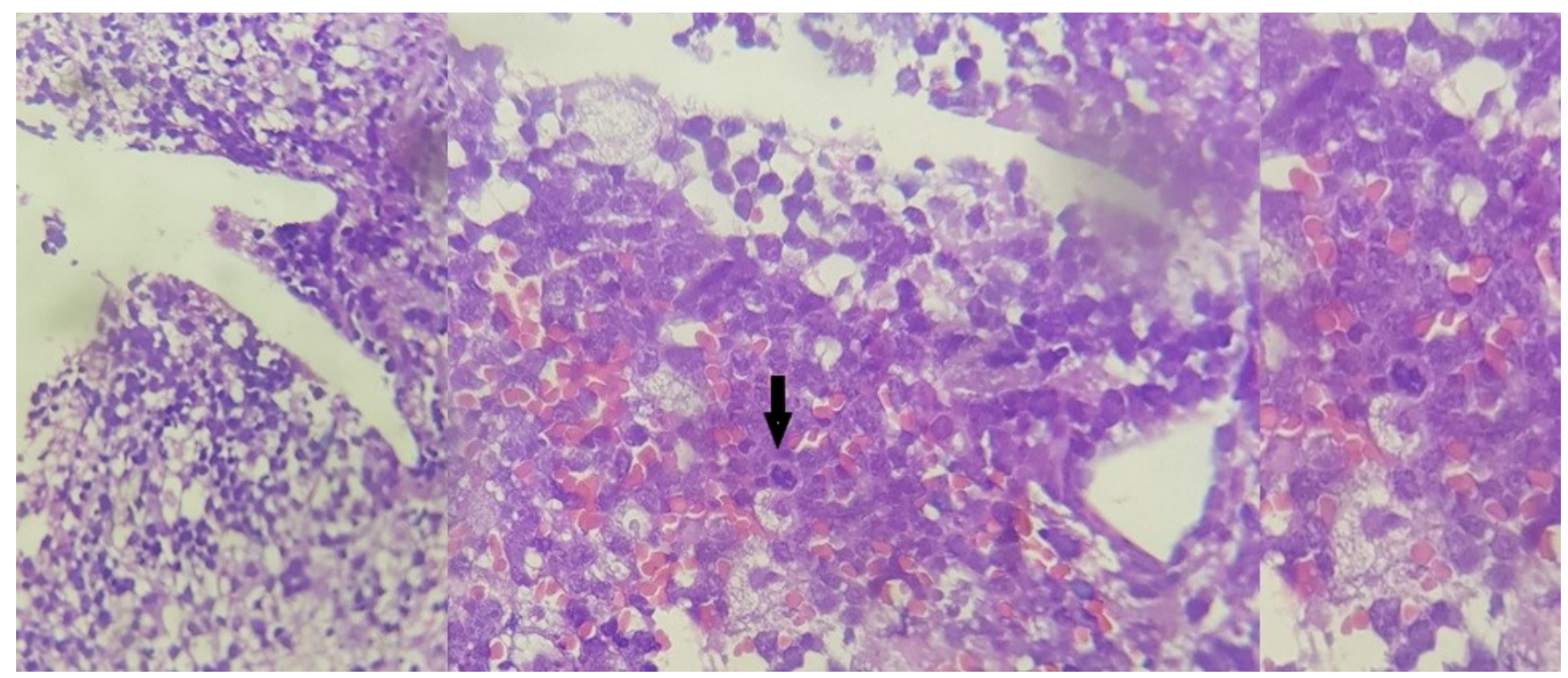

Figure 2. Histopathological examination showed atypical lymphoid cell proliferation.

\section{References}

1. Mark DK, Deva S, Rudolph J, Castellani RE, Morales SG, Reichs ASK, Robert KS. Demyelination as a harbinger of lymphoma: a case report and review of primary central nervous system lymphoma preceded by multifocal sentinel demyelination. BMC Neurol. 2016 May;16-72. https://doi.org/10.1186/s12883-016-0596-1

2. Chiavazza A, Pellerino F, Ferrio A, Cistaro R, Soffietti R, Rudà R. Primary CNS lymphomas: challenges in diagnosis and monitoring. Biomed Res Int. 2018 Jun;2018:3606970. https://doi. org/10.1155/2018/3606970

3. Jian-Giang Lu, Cian O'Kelly, Safwat G, Derek E, Christopher P, Gregg B. Neuroinflammation preceding and accompanying central nervous system lymphoma: case study and literature review. World Neurosurg. 2016 Apr;88:692.e1-692.e8. https://doi.org/10.1016/j. wneu.2015.11.099 